Revista Universo Contábil, ISSN 1809-3337

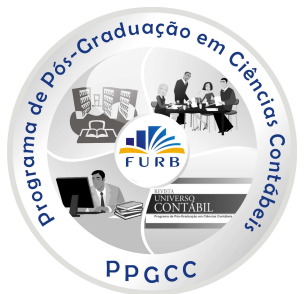

\title{
EL ANÁLISIS DE LOS ESTADOS CONTABLES EN UN ENTORNO DINÁMICO Y GERENCIAL DE LA EMPRESA*
}

\section{ANALYSIS OF FINANCIAL STATEMENTS IN A DYNAMIC AND MANAGEMENT ENVIRONMENT OF THE COMPANY}

\author{
Miguel Diaz Llanes \\ Master en Dirección de Empresas pela Cámara de Comercio de Huelva - España \\ Profesor del Departamento de Dirección de Empresas de la Facultad de Ciencias Empresariales \\ Universidad Pablo de Olavide \\ Endereço: Carretera de Utrera, Kilómetro 1 \\ CEP: 41013 - Sevilla - España \\ E-mail: mdialla@upo.es \\ Telefone: +34954977896
}

\section{RESUMEN}

Con el presente trabajo pretendemos aportar un análisis de los estados contables que tenga en cuenta a la empresa como un ente dinámico donde en cada momento la realidad de la empresa, la competencia y las circunstancias cambian. Para ello aportamos una serie de ajustes que se deben hacer para que los diferentes estados recojan esa realidad de la empresa en cada momento. Así mismo ponemos en relación el análisis vía ratios con la capacidad de la empresa para generar liquidez, solvencia y rentabilidad. Y situamos esa capacidad, principalmente, en los elementos productivos a largo plazo. Así mismo el análisis contable debe ser una herramienta de gestión y de información. Por ello depuramos la información suministrada por los estados contables para conocer en primer lugar la realidad de la empresa analizada y después poder actuar y decidir con las máximas garantías posibles. La reciente aplicación de las normas NIC/NIIF han permitido aproximarnos a esa realidad, pero quedan muchas lagunas que con los actuales planteamientos imposibilitan una toma decisiones adecuada. Como consecuencia de ello llegamos a unos estados contables bien diferentes a los suministrados por la contabilidad, incluso con la adaptación a las nuevas normas internacionales y nacionales de contabilidad. Y por consiguiente variará de forma sensible la liquidez, la solvencia y la rentabilidad de las empresas analizadas.

Palabras claves: Análisis. NIC/NIIF. Ajustes. Fondo de maniobra. Solvencia.

\footnotetext{
Artigo recebido em 10.09.2009. Revisado por pares em 14.12.2009. Reformulado em 17.05.2010. Recomendado para publicação em 20.05.2010 por Ilse Maria Beuren (Editora). Publicado em 30.06.2010. Organização responsável pelo periódico: FURB.
} 


\section{RESUMO}

O artigo pretende apresentar uma análise das demonstrações contábeis, tendo em vista a empresa como uma entidade dinâmica, em que a cada momento a realidade do negócio, a concorrência e as circunstâncias mudam. Assim, oferece uma série de ajustes que devem ser realizados para garantir que as demonstrações representem a realidade da empresa a qualquer momento. Também, relaciona a análise de índices com a capacidade da empresa de gerar liquidez, solvência e rentabilidade, principalmente, quanto aos elementos produtivos a longo prazo. Logo, a análise contábil deve ser uma ferramenta de gestão e informação. Portanto, tratou-se de ajustar as informações oferecidas pelas demonstrações contábeis para conhecer, em primeiro lugar, a realidade da empresa analisada para, em seguida, agir e decidir com a máxima garantia possível. A aplicação recente das NIC/IFRS permitiu uma aproximação maior a essa realidade, mas existem muitas lacunas nas atuais abordagens que impedem uma tomada de decisão adequada. Como resultado, chegou-se a demonstrações contábeis bem diferentes daquelas fornecidas pela contabilidade, mesmo com a adaptação às novas normas internacionais e nacionais de contabilidade. E, portanto, varia de forma significativa a liquidez, solvência e rentabilidade das empresas analisadas.

Palavras-chave: Análise contábil. NIC/IFRS. Ajustes. Capital de giro. Solvência.

\section{ABSTRACT}

The present is intent to present an analysis of the Accounting Statements, having the company as a dynamic entity, in which at every moment the reality of the company, the competition and the circumstances change. Thus, it proposes a series of adjustments that must be done in order to guarantee that the statements represent the reality of the company at every moment. Also, it relates the indexes analysis to the company's ability of generating liquidity, solvency and profitability, mainly regarding the productive elements in the long term. Therefore, the analysis should be an accounting management tool and information. To do so, the information supplied by the accounting statements were adjusted to know, firstly, the reality of the analyzed company to, then, be able to act and decide with the maximum possible guarantees. The recent application of NIC/IFRS allowed greater approximation to this reality, but there many gaps in the current approaches that prevent proper decision taking. As a result, it was found accounting statements very different from the supplied by for the accounting, even with the adjustment to the new international and national standards of accounting. Thus, the liquidity, the solvency and the profitability of the analyzed companies vary significantly.

Keywords: Accounting Analysis. NIC/IFRS. Adjustments. Working capital. Solvency.

\section{INTRODUCCIÓN}

Recordar lo que el director general de una compañía le dijo a una recién licenciada el primer día de trabajo: tenemos muchos contables, pero tú eres algo más; tu eres nuestra analista. Tú papel será ayudarme a tomar decisiones. Algo más de una línea que sintetiza la función del analista. Al final de su trabajo siempre hay una decisión. Pero para que haga bien su trabajo necesita dos cosas. Una, independencia; otra, toda la información o, matizando, toda la información razonablemente posible.

La contabilidad identifica y mide hechos económicos o susceptibles de ser valorados económicamente. Primero debe identificar ¿cuál es? Luego lo tiene que medir, ¿cuánto es? El siguiente paso es comunicar esa información de forma útil, estandarizada y acordada en 
términos amplios. ¿A quién? A los usuarios de la contabilidad que pueden ser internos o externos a la empresa. ¿Para qué? Para formarse un juicio acerca de la empresa y actuar y tomar en consecuencia decisiones.

$\mathrm{Y}$ en ese momento entra en acción el analista. Va a interpretar, a partir de la información de la contabilidad, la realidad de la empresa para que luego las decisiones que se tomen sean beneficiosas para la compañía. Y ese objetivo implica la planificación. ¿Y por qué se planifica y hay un diagnóstico previo? Porque hay que conocer dónde estamos, a dónde vamos y cómo podemos ir hacia nuestros objetivos. Es decir somos vitales para que la empresa pueda decidir y desarrollar sus estrategias.

La inversión, la ampliación, la petición de una ayuda pública o de un préstamo, la negociación con los sindicatos, con proveedores y clientes deberán tener una base muy sólida sustentada en el análisis de los estados contables. Pero hay que indicar que el analista no sólo debe usar y abusar de la información que le proporciona la contabilidad. Si así lo hiciera consideraría a su empresa como un ente aislado de la realidad, del sector, del entorno, de la competencia.

Deberá beber de otras fuentes. De todas aquellas donde existan informaciones que afecten directa o indirectamente a su compañía: registros, archivos, estudios, centrales de balances y también de la prensa especializada, pero con sentido crítico. Por supuesto debe conocer cómo es su empresa en todo aquello que no esté en los libros de contabilidad. El analista debe tener muy presente que la mejor información de la empresa está dentro de la empresa y, no siempre, ni sólo en su contabilidad.

\section{CARACTERÍSTICAS DEL ANÁLISIS DE ESTADOS CONTABLE}

El análisis de estados contables no sigue ni puede seguir unas pautas idénticas, inequívocas, asépticas y objetivas. Quizás porque no hay dos empresas iguales ni dos situaciones económicas iguales. Ésa es la grandeza del analista. El analista debe tener muy presente que la información que maneja nunca tendrá una fiabilidad del $100 \%$. La incertidumbre y la limitación de la información disponible son los elementos con los que trabaja.

Pero éste no es el único problema o limitación: el analista trabaja para un jefe con un perfil, características y manías. En el seno de una empresa con una cultura organizativa determinada, en un sector de dura competencia y en un entorno impredecible. Y siempre trabajará contra reloj. El analista debe proporcionar al que tome las decisiones en la empresa una información útil, con un coste de tiempo y medios razonables, lo más documentada posible y orientada al uso que de ella se vaya a hacer.

No es lo mismo un informe sobre la situación financiera que sobre la rentabilidad; no es lo mismo un análisis para absorber a una empresa que para ser absorbida; no es lo mismo un informe para entrar en nuevos campos de actividad que para negociar con los sindicatos; no es lo mismo un informe para una suspensión de pagos que para pedir una ayuda pública.

No queremos decir que hay que manipular el informe; queremos decir que se emplearán las fuentes informativas, los ratios y las técnicas adecuadas para cada fin. Dentro de la información que maneja el analista están aspectos muy variados como magnitudes macroeconómicas, monetarias, legales, fiscales, sectoriales, de la competencia, el entorno social, político, etcétera.

Las estrategias y decisiones que se tomen están influenciadas (externamente) por el sector en el que se ubica; pero además por su entorno geográfico, social, político y administrativo. Las estrategias están determinadas por diversas cuestiones como el producto o servicio, el grado de integración en la cadena de valor, el grado de diversificación geográfica, el grado de diversificación del sector. Ese conjunto de elementos, junto a los que se producen 
en el interior de la propia empresa son los que van a condicionar las metas y estrategias que se fijen.

\section{LOS ESTADOS CONTABLES}

Constituyen la base de información para el analista a la hora de desarrollar su trabajo. Vamos a plantear este apartado con los nuevos estados que surgen de la aplicación de la normativa NIC-NIIF. Así consideraremos: Balance, Cuenta de Resultados, Estado de Flujo de Efectivo, Estado de Cambios en el Patrimonio Neto y Notas, que en España sigue llamándose Memoria.

\subsection{Balance}

El Balance de Situación informa sobre la situación económica y financiera de una empresa en un momento determinado. La nueva normativa (ESPAÑA, 2008) clasifica al Balance conforme Cuadro 1.

\begin{tabular}{|l|}
\hline ACTIVO \\
\hline Activo No Corriente \\
I Inmovilizado Intangible \\
II Inmovilizado Material \\
III Inversiones Inmobiliarias. \\
IV Inversiones en Empresas del Grupo y Asociadas a Largo Plazo \\
V Inversiones Financieras a Largo Plazo \\
VI Activos por Impuesto Diferido \\
\hline Activo Corriente \\
I Activos no corrientes mantenidos para la venta \\
II Existencias \\
III Deudores Comerciales y Otras Cuentas a Cobrar \\
IV Inversiones en Empresas del Grupo y Asociadas a Corto Plazo \\
V Inversiones Financieras a Corto Plazo \\
VI Periodificaciones \\
VII Efectivo y Otros Activos Líquidos Equivalentes \\
\hline PASIVO \\
\hline Patrimonio Neto \\
I Fondos Propios \\
II Ajustes por Cambio de Valor \\
III Subvenciones \\
\hline Pasivo No Corriente \\
I Provisiones a Largo Plazo \\
II Deudas a Largo Plazo \\
III Deudas con Empresas del Grupo y Asociadas a Largo Plazo \\
IV Pasivos por Impuesto Diferido \\
\hline Pasivo Corriente \\
I Pasivos vinculados con Activos no Corrientes mantenidos para la venta \\
II Provisiones a Corto Plazo \\
III Deudas a Corto Plazo \\
IV Deudas con Empresas del Grupo y Asociadas a Corto Plazo \\
V Acreedores Comerciales y Otras Cuentas a Pagar \\
VI Periodificaciones \\
\hline Cuadro - Batance \\
\hline
\end{tabular}

Cuadro 1 - Balance

\subsection{Cuenta de Pérdidas y Ganancias}

El Estado ó Cuenta de Pérdidas y Ganancias informa sobre la evolución de la empresa en un periodo determinado. La nueva normativa contable (ESPAÑA, 2008) incluye una 
Cuenta de pérdidas y Ganancias muy diferente en su estructura y presentación a la que estábamos acostumbrados. Su nuevo formato es el presentado del Cuadro 2.

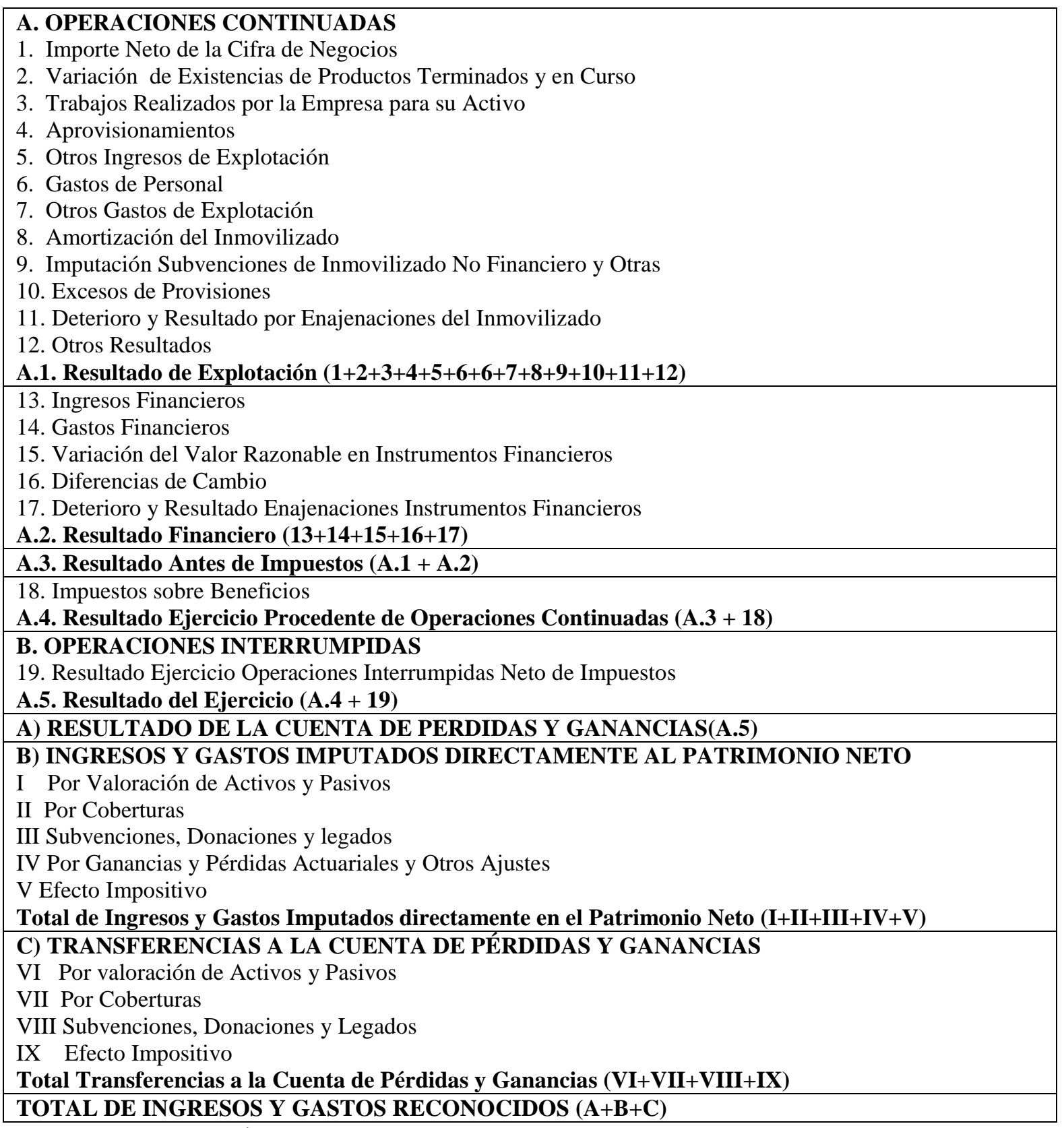

Cuadro 2 - Cuenta de Pérdidas y Ganancias

No podemos pasar por alto la manipulación de los resultados por parte de los ejecutivos, porque muestran unos estados falsos. El analista debe ser consciente de ello y depurarlo para poder llegar a conclusiones correctas y útiles para la toma de decisiones.

Dos son las tácticas ¿legales, permitidas? El alisamiento de resultados consiste en elevar de forma artificial los beneficios en los periodos de crisis y de reducirlos en los de bonanza económica. El objetivo es buscar una estabilidad de los resultados. Con ellos se satisface a muchos accionistas que quieren una renta variable como si fuera fija pero con mayores rendimientos.

Esto lo usan muy a menudo las entidades financieras para no asustar al Banco de España, a la sociedad donde opera y al público en general. Estaría mal visto un excesivo 
incremento de los beneficios así como bajos beneficios o pérdidas. La confianza en estas entidades es fundamental. ¿Cómo se alisan los resultados?

a) adelantando o retrasando el reconocimiento de ciertas partidas de ingresos y gastos;

b) con las dotaciones de amortizaciones y provisiones;

c) con la clasificación de las partidas ordinarias / extraordinarias;

d) pensiones;

e) gastos amortizables;

f) gastos discrecionales;

g) contrataciones de bienes y/o servicios a fin de año;

h) política salarial a fin de año;

i) usando el grupo de empresas con operaciones internas.

Otra de las tácticas es el apuntamiento de tendencia. Empeorar los resultados para obtener altos beneficios en periodos posteriores. También se emplea para justificar despidos. Se suele basar en algún hecho externo creíble y teóricamente incontrolable. Por ejemplo la subida del precio del petróleo, crisis generalizada, sectorial, cambio en la fiscalidad. Se usan un exceso de provisiones para sanear resultados futuros.

\subsection{Memoria}

Este estado tiene un papel decisivo para la mejor comprensión de la contabilidad de una empresa, y por ende es de enorme ayuda para accionistas e inversores. Su mayor utilidad la conseguirá si no se ajusta a unos mínimos legales, sino que aporten todo aquello que complete y complemente la información de los otros estados contables.

La información recogida en la Memoria deberá adaptar su presentación a la exigencia de la claridad. Por tanto, comprensibilidad y claridad son dos requisitos básicos de la información a presentar por este estado contable.

\subsection{Informe de Gestión}

No está recogido en la nueva normativa (ESPAÑA, 2008) como estado contable, ni como documento obligatorio. No obstante su importancia y utilidad para conocer el estado de la sociedad y su futuro es fundamental. Tiene carácter, cualitativo y realiza proyecciones cara al futuro. Su contenido no es cerrado. A modo indicativo debiera incluir, como mínimo, una exposición fiel de la evolución de los negocios y de la situación de la sociedad.

Debe aclarar y/o ampliar la información recogida en Balance y cuenta de Pérdidas y Ganancias, así como aspectos de organización, laborales, remunerativos, etc. También debe incorporar los acontecimientos relevantes para la sociedad ocurridos después del cierre del ejercicio, y que puedan afectarle.

Igualmente debería llevar estimaciones fundamentadas de la posible evolución de la sociedad, poniéndola en relación con el mercado, la legislación, la situación económica general.

\subsection{Informe de Auditoría}

Al igual que el anterior no está aparece en la nueva normativa (ESPAÑA, 2008) como estado contable, ni como documentación obligatoria. Es otro documento que se debe incorporar a la información económica-financiera para una mejor comprensión y seguridad del contenido de las cuentas de una sociedad.

Además constituye, o debería constituir, una fuente de información básica para el analista. Las observaciones de los auditores tienen que ver con la adaptación de la contabilidad a la realidad de la empresa. 


\subsection{Otros Estados Contables}

En esta rúbrica (otros estados contables) vamos a mencionar dos: Información Segmentada y Información Intermedia.

a) Información Segmentada

La segmentación que se puede referir a las líneas de actividad, a los mercados geográficos, a tipos de clientes, etc. El objetivo de la información segmentada es ayudar a los usuarios de los estados financieros a conseguir una mejor apreciación de los resultados y de la posición financiera de la empresa, posibilitando una mejor comprensión de los resultados pasados y una mejor evaluación de las perspectivas futuras.

Lo primero que hay que hacer es definir los segmentos significativos, esto es aquellos que contienen datos relevantes para el usuario. Estos se pueden establecer siguiendo distintos criterios: por área de actividad, por áreas geográficas, por clientes o por entidades legales que integran un conglomerado empresarial.

Sobre qué hay que presentar como información segmentada no hay coincidencia pero existe una percepción de que se debería dar sobre ventas, beneficios, activos y nuevas inversiones por segmento de actividad; y sobre ventas, beneficios y activos por áreas geográficas.

\section{b) Información Intermedia}

Puede ser interesante disponer de una información con una base temporal diferente al año, y que además tenga una regularidad. Por ejemplo semestral, cuatrimestral, trimestral, bimestral, mensual, e incluso quincenal, semanal o diaria. Ello no es óbice para que la anual sea la más usual.

La utilidad de estos informes se ve completada con la dificultad de que al ser mayor el volumen de la información y más corto el periodo de la misma, mayor será el coste del tratamiento y análisis, por lo que hay que determinar la información mínima que deben contener estos informes.

\section{OTRAS FUENTES}

La primera fuente de información contable que tiene el analista se encuentra en la propia empresa. Esta no es sólo la de los libros de contabilidad, sino los múltiples estudios e informes y todas aquellas observaciones, memorandums internos, ideas y estrategias de la dirección general de la compañía.

En principio la máxima información de la empresa está en la propia empresa, y además con el mayor grado de detalle posible. Por otro lado esta información es veraz porque en principio es una información que no sale fuera y que es usada para la gestión del día a día.

También se encuentra información de la empresa en los Registros Oficiales - Registro Mercantil (www.juntadeandalucia.es), CIRBE (www.bde.es) y CNMV (www.cnmv.es). Aquí la información depositada es un mínimo legal exigible y exigido. Son públicos, fiables (dentro de la limitación anterior) y son accesibles informáticamente (y cada vez más). Son informaciones, algunas, por las que hay que pagar.

Más interesantes pueden ser los informes bancarios y los informes periciales solicitados. Su coste compensa por el grado de concreción que tienen. Hay otra línea de información a tener en cuenta y son las informaciones sectoriales de diferentes entidades públicas y privadas. El acceso es fácil, mucha es gratuita, se renueva anualmente y constituyen una herramienta básica a la hora de situar a nuestra empresa en el sector, en el mercado, en la UE. 
No podemos olvidar los informes globales de la situación económica emitidos por el FMI (www.imf.org), la OCDE (www.oecd.org), Eurostat (epp.eurostat.ec.europa.eu), ministerios, consejerías, diputaciones, ayuntamientos. Con ellos podremos situar a empresa en el mundo (Europa, España, Andalucía) y en la economía.

Por último está Internet, una herramienta con un potencial insospechado para la búsqueda de información. Lo más efectivo (¿tiempo?) es la búsqueda personalizada. Lo más práctico es acudir a las páginas web de las propias empresas (siempre dicen cosas buenas, nunca negativas); la de la CNMV (cada vez más completa y exigente), Registro Mercantil, INE y Banco de España. También existe mucha información basura, no útil y sin depurar.

\section{AJUSTAR PARA CONOCER LA REALIDAD}

Ya dijimos en su momento que el trabajo del analista no es algo estructurado, que no hay normas inflexibles ni generales. Por el contrario cada empresa es un mundo distinto. Además existen una multiplicidad de fuentes para conocer: la liquidez, la solvencia y la rentabilidad. Pero todo ello en un entorno sectorial, geográfico, político, económico y social.

Esto hace que no sólo busquemos una información útil para el análisis, sino que además precisamos que sea útil desde diferentes puntos de vista. Para ello se deberá seleccionar toda la información disponible para quedarnos sólo con aquella que sea de interés para nuestro objetivo.

Con ese fin realizaremos ajustes en la información de la empresa (por supuesto en sus estados contables) para sacar lo útil y significativo en un contexto de coste/beneficio/tiempo. Toda la batería de márgenes, rotaciones, ratios, estructura de costes, apalancamiento financiero, apalancamiento operativo, coste de los pasivos, diferentes rentabilidades, deben supeditarse a las exigencias del caso concreto para el que se nos piden el análisis, a la información disponible y al tiempo.

Hay que hacer una observación muy importante. Las eliminaciones y los ajustes que se realicen no afectan a la contabilidad; son anotaciones que realizamos con el único objetivo del análisis que nos ha sido encomendado.

\subsection{Ajustes en el Balance}

A continuación hacemos una selección de casos y situaciones en los que hay que proceder a replantear, ajustar, reclasificar y/o eliminar determinadas partidas del Balance. Buscamos que el punto de partida de nuestro análisis sea el mas exacto posible.

a) Inmovilizado Intangible

No podemos olvidar que en la empresa existen una serie de elementos relacionados con el know how y con las personas que hacen que de dos empresas con idénticos balances una tenga más éxito que otra. Esto hay que estimarlo y cuantificarlo y recogerlo en este apartado. Es cierto que esos elementos no han sido objeto de adquisición mercantilista, pero marcan las diferencias.

Lo que ocurre es que no somos conscientes de su importancia hasta que ocurren hechos como: caída en su cotización o salida de la empresa de elementos esenciales como deportistas, directivos, personas claves por su especialización, resultados de investigación y/o investigaciones en curso.

$\mathrm{Si}$ entendemos que el activo es lo que permite conseguir ingresos, beneficios, inversiones, crecimiento, es evidente que los aspectos reseñados anteriormente son activos. El problema es la valoración. Ésta, aún no siendo tan exacta como el importe de una factura, hay que estimarla y revisarla anualmente para ajustarnos a la realidad. 
Afectará positivamente a cuestiones como la solvencia y la rentabilidad, vía la generación de ventas, beneficios.

b) Inmovilizado Material

Aquí habría que hacer una estimación y un seguimiento con los ajustes pertinentes porque es evidente que el Inmovilizado Material tangible no vale igual en todos los momentos, y no sólo por la amortización. Se da en casos como:

a) caída significativa de su valor en el mercado;

b) cambio significativo del uso que se le da al activo y que suponga para la empresa un incremento/decremento en los ingresos y beneficios generados;

c) aparición en el mercado de unos elementos productivos que mejoran sensiblemente los resultados de los nuestros;

d) cambio estratégico de la compañía;

e) impacto medioambiental de las centrales nucleares y otras instalaciones molestas, insalubres y peligrosas;

f) localización en las proximidades de determinadas actividades que supongan una pérdida del valor de nuestras instalaciones.

Siguiendo con las pérdidas de valor de los activos de una empresa hay que tener presente que las mismas se pueden deber a múltiples circunstancias:

a) los sistemas de uso y/o construcción del activo en cuestión han cambiado y dejan obsoletos a los anteriores (planchas de hormigón y nuevos materiales, uso del pladur en lugar del ladrillo);

b) combustible $\mathrm{u}$ otro elemento que permite el funcionamiento de una máquina que ha caído en desuso;

c) elementos contaminantes en las fábricas. La introducción de medidas correctoras pueden permitir obtener esos subproductos, que se tiran, en mayores cantidades y que se pueden vender como subproductos. Caso del azufre en las industria del refino del petróleo;

d) pérdidas continuas que obligan abandonar el negocio, marginalizarlo, redimensionarlo, reconvertirlo o venderlo a precio de saldo (actividades de nuevas tecnologías);

e) activos que la competencia ha dejado de usar y que para nosotros debe ser una señal para hacer lo mismo;

f) líneas deficietarias en los transportes (nuevos usos, alquiler, disminuir la calidad, cambiar las periodicidades, etc.);

g) venta de productos complementarios en los viajes (bebidas, cascos para la música y el video, tienda a bordo, programa de puntos canjeables);

h) falta de demanda cuando estamos en el proceso de reestructuración (venta a precio de saldo, usos alternativos, esperar a que cambien las condiciones del mercado);

i) máquinas que ya no se usan (venderla a países subdesarrollados).

Todo lo anterior obliga a introducir cambios en el Balance. Los mismos se realizarán teniendo en cuenta estimaciones anuales que permitan una actualización permanente de sus valores.

c) Inversiones en Empresas con Pérdidas

Habría que considerarlo como una Provisión por Riesgos y Gastos por el importe total de la misma. A veces y como medida provisional y conservadora se debería incluir como una inversión financiera con valor negativo. El desembolso realizado o comprometido debería sustituirse por un valor negativo que recogiera el valor real actual de la mencionada inversión. 
d) Parte de Existencias que se Mantiene Ejercicio Tras Ejercicio

Estaríamos ante un componente a largo plazo dentro de una partida a corto plazo. $\mathrm{O}$ como se define en la nueva normativa (ESPAÑA, 2008) existe un componente de no corriente dentro de una rúbrica de corriente. Esta inmovilización es difícil de eliminar pues en todos los negocios se necesitan mantener unos stocks mínimos sea para la producción o para la venta. Nunca podríamos tener las estanterías vacías, pues de lo contrario no podríamos vender nada y habría que esperar a que llegasen nuevas partidas. No es realizable, es una partida no corriente.

e) Incremento Excesivo de la Partida de Existencias de Mercaderías

Puede deberse a una caída de las ventas. En ese caso se debe realizar una provisión por depreciación de existencias en el porcentaje que se estime adecuado. Si por el contrario se debe a una acumulación cara al próximo ejercicio no habría que hacer nada.

f) Parte de Clientes que se Mantiene Ejercicio Tras Ejercicio

Estaríamos ante un componente a largo plazo dentro de una partida a corto plazo. $\mathrm{O}$ como se define en la nueva normativa (ESPAÑA, 2008) existe un componente de no corriente dentro de una rúbrica de corriente. Esta inmovilización es difícil de eliminar pues implicaría unas menores ventas. Probablemente si no se diera crédito a los clientes no se vendería la misma cantidad que se vende.

g) Peso y Calidad de Cada Cliente en el Total de la Cuenta

No es lo mismo tener la cuenta de clientes repartida entre una serie de clientes que nos deben una cantidad similar o que el riesgo se concentre de forma casi exclusiva en un gran cliente. Estamos muy expuestos a ello y habría que adaptar la cuenta de clientes a ese riesgo, mediante los instrumentos que nos permite la contabilidad.

h) Aumento de las Ventas Producido por unas Facilidades Excesivas a Clientes que han Crecido en un Porcentaje Superior al de las Propias Ventas

Se debería llevar ese exceso a clientes de dudoso de cobro y dotar paralelamente la dotación correspondiente.

i) El Saldo de Tesoreria se Incrementa Sensiblemente sobre el de Años Precedentes

Si el mismo provoca que el AC > PC y los plazos de cobros y pagos son similares, se debería traspasar a Activo Extrafuncional. Razonamiento similar cabría hacer de las cuentas de Existencias y de Clientes, y en cierta medida de las Inversiones Financieras Temporales.

\section{j) Parte de Tesoreria que se Mantiene todos los Dias, Meses y Ejercicio Tras Ejercicio}

Estaríamos ante el caso del cambio que se mantiene en las cajas de los comercios y en las de cualquier empresa para atender pagos pequeños, así como las cantidades de seguridad. Si siempre se mantiene, estaríamos ante un componente a largo plazo dentro de una partida a corto plazo. O como se define en la nueva normativa (ESPAÑA, 2008) existe un componente de no corriente dentro de una rúbrica de corriente. Esta inmovilización es difícil de eliminar pues en todos los negocios se necesita mantener una cantidad mínima de efectivo y siempre suele ser la misma. No es realizable, es una partida no corriente.

\section{k) Cuentas Bancarias con Saldo Negativo}

Hay que tener en cuenta que las partidas de banco y de caja nunca pueden ser negativas. En ese caso lo que se ha producido es un préstamo de la entidad financiera a la 
empresa a un tipo de interés del descubierto que es conocido y está recogido en el contrato de la cuenta. Este saldo hay que colocarlo en el pasivo como corriente.

1) Subvenciones Consideradas de Explotación Cuando en Realidad son de Capital

Hay que quitarla de los ingresos de explotación (subvenciones de explotación) y colocarla como Subvenciones de Capital. Esta reubicación se hará por la totalidad de la subvención menos la parte correspondiente al ejercicio. La amortización correspondiente iría a Resultados Extraordinarios. Se ha producido una pérdida por la parte que es a largo plazo (la no amortizada en el periodo).

m) Pólizas que se Renuevan Ejercicio Tras Ejercicio

La póliza de crédito se firman habitualmente a 6 meses o a 12 meses y la renovación suele ser automática y se suele mantener ejercicio tras ejercicio. Por ello estamos con un endeudamiento a largo plazo y no a corto plazo. Por ello hay que considerarlo a largo plazo o como pasivo no corriente.

n) Parte de Proveedores que se Mantiene Ejercicio Tras Ejercicio

Suele ser habitual que las empresas mantengan año atrás año unos saldos de proveedores y acreedores en sus balances. Es una forma de financiación muy habitual. Estamos ante un montante que se mantiene a largo plazo. Puede darse con proveedores y acreedores concretos. Un componente a largo plazo dentro de una partida a corto plazo; o como se define en la nueva normativa (ESPAÑA, 2008) existe un componente de no corriente dentro de una rúbrica de corriente. Esta inmovilización es difícil de eliminar pues implicaría unas menores compras y la reducción de una financiación relativamente barata. Probablemente si no nos dieran crédito nuestros proveedores, no le compararíamos ni nosotros ni otros clientes.

o) Inflación

Se tendría en cuenta en todas las partidas a las que afecta tanto en el activo como en el pasivo y en la cuantía en la que ello ocurra. También tiene su impacto en la Cuenta de Pérdidas y Ganancias.

Y habría muchos mas casos.

\subsection{Ajustes en la Cuenta de Pérdidas y Ganancias}

El Beneficio Neto es útil como una medida del pasado y como una manera de conocer la rentabilidad de las operaciones de una compañía. Por otra parte es la variable más importante para conocer el valor de las acciones de una sociedad. Es una manera razonable de medir el valor añadido de una empresa durante un periodo determinado y del valor probable que puede incorporar en el futuro.

La conexión beneficios - valor añadido puede desaparecer por:

a) la inclusión en los beneficios de ingresos, gastos, ganancias y pérdidas que no se esperan que ocurra en el futuro (Beneficios No Recurrentes);

b) unos inadecuados sistemas de contabilidad para medir el valor añadido de forma fiel y fidedigna de las actividades ordinarias de la empresa durante el periodo de referencia o al que se refiere (Cálculo de los Beneficios);

c) la oportunidad (o posibilidad) de la dirección para manejar (gestionar), o quizás manipular, el nivel (cantidad) o la tendencia de los beneficios en su propio provecho.

La evaluación de los beneficios, teniendo en cuenta la presencia de uno o varios de estos factores, es lo que nos lleva a hablar de Calidad de los Beneficios. A los tres aspectos 
habría que añadir dos más: Beneficios permanentes (a lo largo del tiempo) y los transitorios (los que se consiguen una vez).

A modo de resumen se puede asegurar que hay muchos factores que pueden afectar la calidad de los beneficios como un indicador de los beneficios que se puedan alcanzar en el futuro. Entendiendo la naturaleza y la extensión de los ajustes necesarios para conocer los beneficios y usarlos como elemento predictivo el analista debe conocer el sector, su compañía y estrategia, y los estados contables.

Es una tarea que incorpora más arte que ciencia (la ciencia se le supone) y que requiere altas dosis de sentido crítico por parte del analista y de experiencia. Además de los ajustes que dimanan de lo expuesto anteriormente tendríamos que tener en cuenta lo siguiente:

a) Aumento de las Ventas Producido por unas Facilidades Excesivas a Clientes que han Crecido en un Porcentaje Superior al de las Ventas

Se debería llevar ese exceso a clientes de dudoso de cobro y dotar paralelamente la dotación correspondiente.

\section{b) Ventas Concentradas en unos Pocos Clientes}

Esto constituye un riesgo a valorar por las empresas desde el momento en que las ventas no están garantizadas y quedamos a expensas de lo que decidan otros. Hay que introducir algún tipo de provisión en función del porcentaje que suponga sobre el total, forma de pago.

c) Compras Concentradas en unos Pocos Proveedores

Es un caso similar al anterior, y se complica porque puede estrangular nuestra capacidad de venta y/o de producción. Habría que introducir alguna cuenta que recogiera ese riesgo, en función del porcentaje que suponga sobre el total, forma de pago.

Y habría muchos mas casos.

\section{LOS AJUSTES Y SUS REPERCUSIONES}

Antes del análisis de los estados contables hay que realizar los anteriores ajustes. El ajuste de las partidas de activo y de pasivo obligará a reelaborar las correspondientes de Patrimonio Neto, Pasivo o Activo. Igual, cuando se tocan las cuentas del Estado de Pérdidas y Ganancias. Reelaborar las partidas de ingresos y de gastos conlleva modificaciones en el beneficio de la empresa y también en el Patrimonio Neto.

Los ajustes son especialmente importantes cuando supongan cambios en el Patrimonio Neto (Análisis de Solvencia) y en los Recursos Propios (Análisis de Rentabilidad). El analista deberá depurar los efectos en la contabilidad de prácticas de maquillaje y/o manipulación de los estados contables. Estas actuaciones se pueden producir de diferentes maneras:

a) clientes dudosos;

b) amortizaciones lentas o aceleradas;

c) usar ingresos atípicos para compensar los ordinarios;

d) provisiones de pasivo que se hacen de activos;

e) valoración de los stocks;

f) uso inadecuado de las reservas;

g) no provisionar ciertos riesgos y gastos.

Por otro lado, a la hora de hacer comparaciones con otras empresas, debemos tener en cuenta y hacer los ajustes pertinentes en los casos de que la fecha de cierre de la contabilidad sea diferente.

La solución ante la variedad de posibles circunstancias hay que buscarla en una salida que dé respuesta al mayor número de interrogantes sin crear nuevos problemas. Por ejemplo 
utilizar informes trimestrales, promediar las cifras del Balance y de la Cuenta de Pérdidas y Ganancias o simplemente prescindir del hecho extraordinario. Una vez más el Informe del Auditor y la Memoria pueden aportar una información muy útil al analista.

\section{INCIDENCIA EN LA LIQUIDEZ, SOLVENCIA Y RENTABILIDAD}

El análisis contable se centra en tres aspectos principales (RIVERO TORRE, 2002):

a) liquidez es la capacidad que tiene una empresa o institución para satisfacer sus deudas inmediatas. Las partidas que lo permiten son caja y bancos. Y también a un plazo superior (clientes y existencias). A efectos de cálculo se extiende hasta el año;

b) solvencia es la capacidad que tiene una empresa o institución para satisfacer sus deudas a largo plazo (más de 1 año). Las partidas que lo posibilitan son las consideradas como Activo No Corriente, pero también podemos usar el Activo Corriente;

c) rentabilidad es la capacidad que tiene la empresa para generar beneficios con el uso de sus bienes y derechos.

Ahora viene el momento de hacer las precisiones. La primera es que la liquidez es la solvencia a corto plazo. Además de las disponibilidades de tesorería existen ciertos activos financieros que permiten la disposición inmediata (fondos, certificados) gracias a las facilidades que brindan Internet y a la competencia entre las entidades financieras.

En cuanto a la capacidad del Activo Corriente para satisfacer las deudas corto plazo hay que matizar que una parte del activo a corto es realmente a largo (clientes, existencias), con lo que hay que depurar y ajustarlo. Por otro lado siempre el Pasivo Corriente ha de ser abonado antes del año, pero existe la posibilidad de la renegociación

En cuanto a la Rentabilidad hay que decir que es un concepto extraño pues la idea la podemos tener todos, pero hay que precisarla, concretarla, darle apellidos. Rentabilidad de qué, sobre qué, para qué y cuándo y dónde. Todo ello me va a condicionar la forma de medir la rentabilidad. Además será siempre un dato relativo. La rentabilidad no es volumen (el volumen es el beneficio) es relación, es comparación.

El apellido se lo vamos a dar cuando sepamos qué tipo de rentabilidad necesito conocer. Porque si bien el beneficio está definido por la diferencia entre los ingresos y los gastos necesarios para conseguirlo, la situación cambiará mucho. Porque la rentabilidad incorpora una idea: Beneficio, pero con qué medios y, sobre todo, con cuántos medios.

En este momento estamos introduciendo los distintos tipos de rentabilidades y su cálculo. No es lo mismo conseguir unos beneficios de 1.000 con unos fondos propios de 500 que con unos de 50.000. No es lo mismo conseguir unos beneficios de 1.000 con un activo de 2.000 que con uno de 100.000. No es lo mismo conseguir unos beneficios de 1.000 con unas ventas de 200 que con unas ventas de 1.000.000. No es lo mismo conseguir unos beneficios de 1.000 en el sector de la alimentación que en el de la publicidad. No es lo mismo conseguir unos beneficios de 1.000 hoy o dentro de 2 años. No es lo mismo conseguir unos beneficios de 1.000 en momentos de crisis que en momentos de bonanza. No es lo mismo conseguir unos beneficios de 1.000 en Vietnam que en España.

\subsection{Liquidez}

Si definimos la Liquidez de una empresa como su capacidad de afrontar sus deudas a corto plazo, tenemos varias formas de medirla. La primera de ella es a través del Fondo de Maniobra. Lo podríamos definir, y calcular, como la diferencia entre Activo Corriente (circulante o a corto plazo) y Pasivo Corriente (circulante o a corto plazo). Hay un consenso generalizado en considerar el corto plazo hasta 1 año. 
Para calcular el impacto deberemos depurar las cuentas de tesorería, cliente y existencias a lo que es corto plazo en sentido estricto, esto es lo que se puede disponer sin que se produzcan alteraciones en el funcionamiento de la empresa.

Del lado del activo minoraremos la tesorería en lo que permanece fijo año tras año por motivos de operativa. También reduciremos las existencias en lo que hayamos definido como stock de seguridad. Por último minoraremos los clientes en el importe que permanece fijo año tras año como consecuencia de la política comercial que tenga nuestra empresa y/o el sector en el que trabajamos. También deberíamos estimar y ajustar las partidas relacionadas con clientes por excesivas facilidades comerciales o sobre exposición a ciertos clientes.

Del lado del pasivo pasaremos las pólizas de crédito que se renuevan año tras año a deudas a largo plazo. Igual haremos con la cuenta de proveedores. Por último ajustaremos las subvenciones de capital mal contabilizadas.

$\mathrm{Si}$ usamos cocientes, en lugar de Fondo de Maniobra haremos las mismas consideraciones y aplicaremos los cocientes típicos:

Ratio Circulante $=$ Activo Corriente $/$ Pasivo Corriente.

Ratio Quick $=($ Tesorería + Inversiones Financieras Temporales + Clientes $) /$ Pasivo Corriente

Ratio Cash $=($ Tesorería + Inversiones Financieras Temporales $) /$ Pasivo Corriente

Por último veremos el impacto en las Rotaciones y en los Periodos Medios de Maduración. Recordar que estos índices no son iguales en todos los sectores y tienen mucho que ver con las prácticas comerciales.

\subsection{Solvencia}

Definiremos la Solvencia como la capacidad que tiene una empresa para afrontar sus deudas a largo plazo y tenemos varias formas de calcularla. Por ejemplo mediante la:

Solvencia Global Garantía = Activo Real / Financiación Ajena

Todas mis deudas a devolver las podré pagar con todo mi activo. Puede ser útil saber cuál es mi independencia financiera, esto es en qué medida mis fondos propios pueden hacer frente a lo que debo:

Independencia Financiera $=$ Fondos Propios $/$ Pasivo Total

Lo podemos ver en términos de dependencia financiera:

Dependencia Financiera $=$ Exigible Total $/$ Pasivo Total

Y quizás pueda pagar mis deudas con el activo extrafuncional:

Activos Extrafuncionales / Exigible Total

Aquí tendré que considerar el componente del activo intangible no contabilizado por culpa de las actuales normas contables (ESPAÑA, 2008) y a los que hicimos referencia anteriormente. Igualmente deberíamos reducir los activos materiales cuyo uso o circunstancias han cambiado negativamente, y en caso positivo hacer el ajuste contrario. También minoraremos las inversiones en empresas con pérdidas. También deberíamos estimar 
y ajustar las partidas relacionadas con clientes por excesivas facilidades comerciales o sobre exposición a ciertos clientes.

\subsection{Rentabilidad}

Podemos definir la Rentabilidad como la capacidad de la empresa para generar beneficios que redundan en futuras inversiones, inferiores deudas, mas beneficios, mayor crecimiento. Existen muchas formas de calcular la rentabilidad. Nos centraremos, al objeto de este trabajo: Rentabilidad Económica y Rentabilidad Financiera.

\section{a) Rentabilidad Económica}

La Rentabilidad Económica vamos a obtener relacionando el beneficio alcanzado con los medios materiales (económicos) de los que dispongo, esto es con el activo real: Beneficios antes de Impuestos e Intereses / Activo Real.

Se le conoce por sus siglas en inglés ROA (Return Over Assets). Hay que indicar que si en la empresa en cuestión su activo tiene oscilaciones, o se maquilla a fin de año, más exacto y correcto sería tomar el Activo Total Medio (ATM), muy usado en las entidades financieras.

El ROA mide de forma separada las actividades operativas y las de inversión, de las de financiación. De esta manera con el ROA puedo comparar dos o más empresas con diferentes pasivos y con estructuras de pasivo distintas. La fórmula general del ROA:

ROA $=$ Beneficios / Activo

L la puedo desglosar, a su vez, en dos cocientes: Beneficio / Ventas y Ventas / Activo. Este desglose me da una información añadida pues me puede identificar donde está la mejoría o empeoramiento del ratio. El primero me recoge el margen de mi actividad. El segundo la rotación de mis ventas.

Para un cálculo del ROA adaptado a la realidad debemos tener presente las consideraciones realizadas en las páginas anteriores sobre el Inmovilizado Intangible, el Inmovilizado Material. Las inversiones en empresas con pérdidas, la dependencia de Clientes, el exceso de facilidades a Clientes, las subvenciones mal contabilizadas, y a mas largo plazo los aspectos vinculados a la concentración en pocos clientes y/o en pocos proveedores.

b) Rentabilidad Financiera

La Rentabilidad Financiera calcularemos relacionando los Beneficios antes de Impuestos con el Patrimonio Neto o capitales propios:

$\mathrm{ROE}=$ Beneficios antes de Impuestos / Patrimonio Neto.

Se le conoce por sus siglas en inglés ROE (Return On Equity). Es un clásico en el análisis de empresas y muy usado en la información de los analistas bursátiles (JIMENEZ CARDOSO; GARCÍA-AYUSO COVARSÍ; SIERRA MOLINA, 2002).

Para calcular la rentabilidad financiera real tendremos en cuenta los aspectos vinculados a las subvenciones mas contabilizadas y en una esfera mas amplia a unos ingresos quizás inflados por excesiva facilidades a lo clientes.

\section{MATIZACIONES A LOS RATIOS CONTABLES}

Podemos inventar y elaborar cientos de ratios, pero la primera condición es que sean útiles, que sirvan para algo, y como premisa deben relacionar magnitudes entre las que exista una 
relación económica-financiera. También deben explicar lo que ha pasado, y tener un cierto grado de predictivo (STICKNEY; BROWN, 2004).

El ratio es una herramienta básica pues reduce el número de variables a las realmente útiles, porque permiten ver la evolución de nuestra empresa, porque podemos realizar comparaciones sectoriales y porque al ser aceptados a nivel internacional, las valoraciones son más amplias e interesantes (en todos los países se habla el mismo idioma de ratios).

Pero no son la panacea pues pierden utilidad cuando se comparan dos empresas con una dimensión muy diferente, también hay que tener en cuenta la manera de calcularlo y para qué quiero el ratio. Es decir está muy condicionado por las peticiones que nos haga la dirección sobre el uso y el objetivo de los ratios y del informe que le vamos a suministrar.

La comparación de ratios en diferentes periodos de tiempo permite analizar la influencia de los cambios en la economía, en el sector y en la empresa. Para valorar correctamente estos cambios el analista debe conocer si se han producido modificaciones significativas en los productos que vende, en sus clientes, proveedores, políticas comerciales.

También si se ha producido un hecho de alcance como una absorción, venta de divisiones, cambio en la política general de la compañía o incluso en sus sistemas contables. Además si alguna disposición normativa ha obligado a cambiar la estructura del activo o de la práctica contable (por ejemplo cuando el Banco de España exigió una política de provisiones más dura).

Cuando procedamos a comparar nuestra compañía con otras o con el sector debemos analizar hechos relevantes, las características del sector, el grado de concentración del mercado, conocer el nivel de desviación respecto a la media, el momento del ciclo de vida en el que nos encontramos, la forma en que las empresas realizan el cálculo de sus ratios.

\subsection{Estructura Patrimonial}

Este tipo de análisis pretende conocer si una empresa es más sólida en su estructura. Esto va en consonancia con el tipo de empresa (grande o pequeña), el sector al que pertenezca y con las políticas de gestión. Ello quiere decir que no existe una estructura ideal y única. Lo que sí es interesante es conocer la variación de las estructuras temporales dentro de la empresa y en comparación con otras para sacar conclusiones.

Para ello usaremos dos tipos de herramientas: los porcentajes verticales y los porcentajes horizontales. Los primeros me permiten conocer la estructura y composición de la compañía (qué parte es Activo No Corriente, qué es Activo Corriente, qué son fondos propios, exigible). Los segundos me posibilitan ver la evolución en el tiempo. Y ambos me dan una idea bastante exacta de dónde está la empresa y hacia dónde va (GOXENS ORENSANZ; GAY SALUDAS, 2002).

Además, estos índices verticales y horizontales coadyuvan a la comparación entre empresas, pero bajo las premisas del primer párrafo. Aquí ajustaremos las distintas masas del activo, del pasivo y del patrimonio neto a las observaciones realizadas en las páginas anteriores.

\subsection{Resumen}

A modo de colofón incluimos un cuadro resumen (Cuadro 3) del impacto de los ajustes propuestos en la liquidez, solvencia y rentabilidad. 


\begin{tabular}{|l|l|l|l|l|l|}
\hline Cuenta & $\begin{array}{l}\text { Movimi- } \\
\text { ento }\end{array}$ & Consideración & Liquidez & Solvencia & $\begin{array}{l}\text { Rentaba- } \\
\text { lidad }\end{array}$ \\
\hline Inmovilizado Intangible & Aumenta & Más Activo No Corriente & No & Sube & Baja \\
\hline Inmovilizado Material & Baja & Menos Activo No Corriente & No & Baja & Sube \\
\hline $\begin{array}{l}\text { Inversión en empresas con } \\
\text { pérdidas }\end{array}$ & Baja & Menos Activo No Corriente & No & Baja & Sube \\
\hline Existencias que se mantienen & Baja & Más Activo No Corriente & Baja & No & No \\
\hline Aumento excesivo Existencias & Baja & Más Activo No Corriente & Baja & No & No \\
\hline Clientes que se mantienen & Baja & Más Activo No Corriente & Baja & No & No \\
\hline Dependencia de Clientes & Baja & Menos Activo Corriente & Baja & Baja & Sube \\
\hline $\begin{array}{l}\text { Aumento en Ventas facilidades } \\
\text { Clientes }\end{array}$ & Baja & Menos Activo Corriente & Baja & Baja & Sube \\
\hline $\begin{array}{l}\text { Aumento en Ventas facilidades } \\
\text { Clientes }\end{array}$ & Baja & Menos Ingresos & No & No & Baja \\
\hline Aumento excesivo Tesorería & Baja & Más Activo Extrafuncional & No & No & No \\
\hline Tesorería que se mantiene & Baja & Más Activo No Corriente & Baja & No & No \\
\hline Banco saldo negativo & No & Sería Pasivo Corriente & No & No & No \\
\hline Subvenciones mal consideradas & Baja & Menos Ingresos & No & No & Baja \\
\hline Pólizas que se renuevan & Baja & Más Pasivo No Corriente & Sube & No & No \\
\hline $\begin{array}{l}\text { Proveedores que se mantienen } \\
\text { Clientes }\end{array}$ & Baja & Más Pasivo No Corriente & Sube & No & No \\
\hline Cuadras en pocos & Baja & Menos Activo Corriente & Baja & No & Baja \\
\hline
\end{tabular}

Cuadro 3 - Impacto de los Ajustes en el Análisis Contable

\section{EJEMPLO PRÁCTICO}

Sea una empresa cuyo balance es el presentado en el Cuadro 4.

\begin{tabular}{|ll|ll|}
\hline ACTIVO & & PASIVO & \\
\hline Inmovilizado & 5.000 & Capital+Reservas & 1.000 \\
Existencias & 20.000 & Beneficios & 5.000 \\
Clientes & 10.000 & Deudas a L. P. & 15.000 \\
Bancos & 3.000 & Póliza Crédito & 5.000 \\
Caja & 2.000 & Acreedores & 14.000 \\
\hline Total Activo & 40.000 & Total Pasivo & 40.000 \\
\hline
\end{tabular}

\section{Cuadro 4 - Balance Ejemplo}

Hemos calculado en el Cuadro 5 el Fondo de Maniobra, el Índice de Circulante, el de Solvencia, el ROE y el ROA.

\begin{tabular}{|lc|}
\hline Fondo Maniobra & 16.000 \\
Índice Circulante & $184 \%$ \\
Índice Solvencia & $85 \%$ \\
ROE & $500 \%$ \\
ROA & $12,50 \%$ \\
\hline
\end{tabular}

\section{Cuadro 5 - Fondo de Maniobra y Índices}

Consideremos ahora las siguientes circunstancias: 
a) existen en la empresa unos elementos intangibles vinculados al personal y no contabilizados por valor de 25.000 ;

b) la empresa trabaja con unas existencias mínimas coincidentes con el stock de seguridad;

c) la política de ventas de la empresa ha llevado a los directivos de que es imposible reducir los clientes en más de la mitad de su saldo;

d) la cuenta de caja tiene en su totalidad el calificativo de mínimo vital;

e) la póliza de crédito se renueva todos los años.

Realizar los ajustes pertinentes en el Balance y recalcular los diferentes índices:

En el Cuadro 6 tiene los ajustes pertinentes en el Balance.

\begin{tabular}{|ll|ll|}
\hline ACTIVO & & PASIVO & \\
\hline Inmovilizado & 57.000 & Capital+Reservas & 26.000 \\
Existencias & 0 & Beneficios & 5.000 \\
Clientes & 5.000 & Deudas a L. P. & 20.000 \\
Bancos & 3.000 & Póliza Crédito & 0 \\
Caja & 0 & Acreedores & 14.000 \\
\hline Total Activo & 65.000 & Total Pasivo & 65.000 \\
\hline
\end{tabular}

Cuadro 6 - Nuevo Balance

El inmovilizado se vería incrementado en el intangible, la totalidad de las existencias, la mitad de los clientes y el valor íntegro de la caja. Por otro lado hemos llevado a Reservas el montante calculado del intangible. En el pasivo la totalidad de la póliza pasa a largo plazo.

Los nuevos índices serían los en el Cuadro 7.

\begin{tabular}{|lc|}
\hline Fondo Maniobra & -6.000 \\
Índice Circulante & $57,00 \%$ \\
Índice Solvencia & $52,31 \%$ \\
ROE & $3,85 \%$ \\
ROA & $1,54 \%$ \\
\hline
\end{tabular}

Cuadro 7 - Nuevos Índices

Desde la óptica de la Liquidez observamos que la empresa no sólo no puede atender a sus pagos sino que está en suspensión de pagos real. Por otro lado la Solvencia ha mejorado al tenerse en cuenta los intangibles. Por último la Rentabilidad empeora notablemente tanto la financiera como la económica.

Quizás la realidad de la empresa dista mucho de la que recoge la contabilidad con los criterios actuales. Ah, los bancos no entiende la solvencia si no es en términos tangibles.

\section{CONCLUSIONES}

Con el presente trabajo pretendemos aportar un análisis de los estados contables que tenga en cuenta a la empresa como un ente dinámico donde en cada momento la realidad de la empresa, la competencia y las circunstancias cambian. Para ello aportamos una serie de ajustes que se deben hacer para que los diferentes estados recojan esa realidad de la empresa en cada momento. Así mismo ponemos en relación el análisis vía ratios con la capacidad de la empresa para generar liquidez, solvencia y rentabilidad. Y situamos esa capacidad, principalmente, en los elementos productivos a largo plazo. 
Señalamos las siguientes conclusiones:

a) el objeto final del análisis contable es la toma de decisiones;

b) debe ampliar sus fuentes a información contable y no contable dentro y fuera de la empresa;

c) el balance debe re-estructurarse porque existen elementos a corto plazo que en realidad son y se comportan como largo plazo, y viceversa;

d) se debe analizar e interpretar la evolución de las diferentes partidas y realizar ajustes y reclasificaciones;

e) hay que relacionar las partidas de Pérdidas y Ganancias con sus equivalentes de Activo y Pasivo, pues pueden encubrir problemas en el Activo y en el Pasivo;

f) la liquidez, para nosotros, no es la diferencia entre Activo a corto plazo menos Pasivo a corto plazo, sino la capacidad que tiene la empresa para generar fondos, $\mathrm{y}$ esta capacidad se encuentra principalmente en el Activo Fijo (el que proporciona continuidad y estabilidad);

g) la solvencia, para nosotros, es la capacidad de la empresa para generar recursos que permita afrontar todo tipo de gastos a Medio y Largo plazo. Nunca lo podemos medir por diferencia entre Activo Real y Pasivo Exigible, pues si vendo Activo o lo reduzco, no podemos seguir funcionando;

h) la rentabilidad, para nosotros, es al capacidad de la empresa para generar beneficios (igual a recursos) para futuras inversiones, producciones, ventas, márgenes, para seguir funcionando;

i) la estructura patrimonial, para nosotros, está íntimamente relacionada con las políticas y estrategias de la empresa y con la competencia.

La empresa es un ente vivo, en cambio y en adaptación permanentes. La troma de decisiones tiene que estar sustentada en un análisis contable dinámico y que contemple a la empresa en funcionamiento, evolución y adaptación. Tiene que ser un análisis que mire al futuro y con datos en clave de futuro. Nunca debe quedarse en el pasado y en explicar que es lo que ya ha sucedido. En su lugar tiene que permitir y explicar, qué pede suceder en el futuro.

El análisis contable debe ser una herramienta de gestión y de información. Por ello depuramos la información suministrada por los estados contables para conocer en primer lugar la realidad de la empresa analizada y después poder actuar y decidir con las máximas garantías posibles.

La reciente aplicación de las normas NIC/NIIF han permitido aproximarnos a esa realidad, pero quedan muchas lagunas que con los actuales planteamientos imposibilitan una toma decisiones adecuada. Como consecuencia de ello llegamos a unos estados contables bien diferentes a los suministrados por la contabilidad, incluso con la adaptación a las nuevas normas internacionales y nacionales de contabilidad. Y por consiguiente variará de forma sensible la liquidez, la solvencia y la rentabilidad de las empresas analizadas.

\section{REFERENCIAS}

ASOCIACIÓN ESPAÑOLA DE CONTABILIDAD Y ADMINISTRACIÓN DE EMPRESAS (AECA). La contabilidad de gestión como instrumento de control. Disponible en: <http://www.aeca.es/comisiones/comisionpg.htm>. Acceso en: 10 ago. 2009.

\section{CENTRAL DE INFORMACIÓN DE RIESGOS EL BANCO DE ESPAÑA (CIRBE). Portal del Cliente Bancario. Disponible en: <http://www.bde.es/clientebanca/entidades/sobreque.htm>. Acceso en: 06 maio 2009.}

COMISIÓN NACIONAL DEL MERCADO DE VALORES (CNMV). Legislación española. Disponible en: < http://www.cnmv.es/Portal/legislacion/legislacion/reciente.aspx > . Acceso en: 08 jul. 2009. 
ESPAÑA. Nuevo Plan General de Contabilidad. 2 ed. Madrid: Ediciones Pirámide, 2008. FONDO MONETARIO INTERNACIONAL (FMI). Organization \& Finances. Disponible en: < http://www.imf.org/external/about/orgfin.htm>. Acceso en: 23 maio 2009.

GABINETE DE ESTATÍSTICAS DA UNIÃO EUROPEIA (EUROSTAT). Headline indicators.

<http://epp.eurostat.ec.europa.eu/portal/page/portal/sdi/indicators>. Acceso en: 03 jun. 2009.

GOXENS ORENSANZ, María Ángeles; GAY SALUDAS, José María. Análisis de estados contables: diagnóstico económico-financiero. Madrid: Prentice Hall, 2002.

JIMENEZ CARDOSO, Sergio M.; GARCÍA-AYUSO COVARSÍ, Manuel; SIERRA MOLINA, Guillermo J. Análisis financiero. Madrid: Ediciones Pirámide, 2002.

JUNTA DE ANDALUCÍA. Publicaciones. Disponible en: <http://www.juntadeandalucia.es/temas/personas/administracion/normativa.html>. Acceso en: 10 ago. 2009.

ORGANIZAÇÃO PARA A COOPERAÇÃO E DESENVOLVIMENTO ECONÓMICO (OCDE). Documents. Disponible en: <http://www.oecd.org/publications/0,3353,en_2649_201185_1_1_1_1_1,00.htmll>. Acceso en: 10 ago. 2009.

RIVERO TORRE, Pedro. Análisis de balances y estados complementarios. Madrid: Ediciones Pirámide, 2002.

STICKNEY, Clyde P.; BROWN Paul R. Financial reporting and statement analysis: a strategic perspective. Orlanado, Florida (EUA): Harcourt Brace College Publishers, 2004. 